\title{
Research on Diabetes by Respiration Patterns Access
}

\author{
R. Kishore Kanna, S.Geetha, T.Manoj Prasath, F.Emerson Solomon
}

\begin{abstract}
Diabetes is a standout amongst the most widely recognized illness that influences numerous people. Diabetes can be alluded to as an interminable malady portrayed by abnormal amounts of sugar (glucose) in the blood. Customarily diabetes is recognized by taking blood tests, however this strategy is difficult. Henceforth by planning an Electronic nose diabetes can be recognized with just breathed out breath tests dependent on biomarkers content. In this paper a minimal effort, non-intrusive framework for distinguishing diabetes is proposed. For this reason, number of breath tests were gathered from typical and diabetic patients to distinguish the biomarker content in breath. E-Nose is utilized to identify the diabetes utilizing unpredictable natural mixes from inhale tests. E-Nose is planned utilizing Arduino MEGA 2560 and gas sensors inserted in Nose cover. In the wake of gathering simple sign from the gas sensor, simple sign is changed over into computerized values for highlight extraction and determination. In highlight extraction fitting qualities were chosen. At that point preparing is finished utilizing ANN and qualities are tried for exactness. The outcomes can be seen in PC[1].
\end{abstract}

Keywords: ENOSE, BIOMAKERS, DIABETES,ARDUINO, RESPIRATION.

\section{I.INTRODUCTION}

Diabetes has been risen as India's seventh greatest reason for early demise in 2016, up from eleventh in 2005, demonstrates information from Institute of Health Metrics and Evaluation. Around $47.3 \%$ of India's 70 million diabetics are undiscovered in which diabetes pervasiveness is higher in prosperous states like Tamil nadu, Kerala, Chandigarh and Maharashtra. Diabetes can be alluded to as a ceaseless sickness portrayed by abnormal amounts of sugar(glucose) in the blood. Customarily diabetes is recognized by taking out the blood tests from the body, however this strategy is difficult and awkward. Thus, by structuring an Electronic nose the diabetes can be distinguished with the breathed-out breath tests from the patient dependent on the biomarkers content. This can be elective technique for distinguishing diabetes. E-Nose investigations the breath tests to get data from biomarkers that is gotten from the breath. E-nose is a progressive item since it is minimal effort, compact, simple to deal with, nonobtrusive system. E-Nose is proposed to recognize the diabetes by dissecting breath tests from human to separate

Revised Manuscript Received on July 18, 2019.

R. Kishore Kanna, Dept of Biomedical Engineering, BIHER, Bharath Instituteof Higher Education and Research, Chennai, Tamil Nadu, India.

S.Geetha, Dept of Biomedical Engineering, BIHER, Bharath Instituteof Higher Education and Research, Chennai, Tamil Nadu, India

T.Manoj Prasath, Dept of Biomedical Engineering, BIHER, Bharath Instituteof Higher Education and Research, Chennai, Tamil Nadu, India

Dr.F.Emerson Solomon, Dept of Biomedical Engineering, BIHER, Bharath Instituteof Higher Education and Research, Chennai, Tamil Nadu, India ordinary and diabetic patient. This methodology is finished by building an E-nose, gathering the simple qualities and changing over them to advanced qualities. Highlight extraction and determination is accomplished for choosing proper qualities, testing is performed for acquiring precision[2].

\section{METHODOLOGY}

E-Nose is intended for recognition of diabetes by breaking down the biomarkers from breath tests that is caught by the gas sensors which are inserted in the E-Nose veil and associated with the microcontroller Arduino MEGA 2560. Outline of the framework can be found in Figure 1 and the framework configuration can be found in Figure 2.

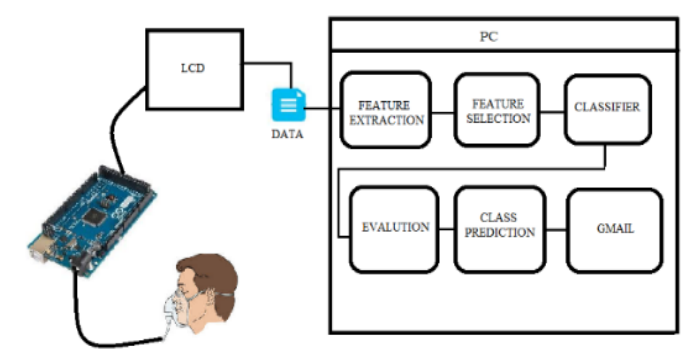

Figure 1. Blueprint of E-Nose

System design

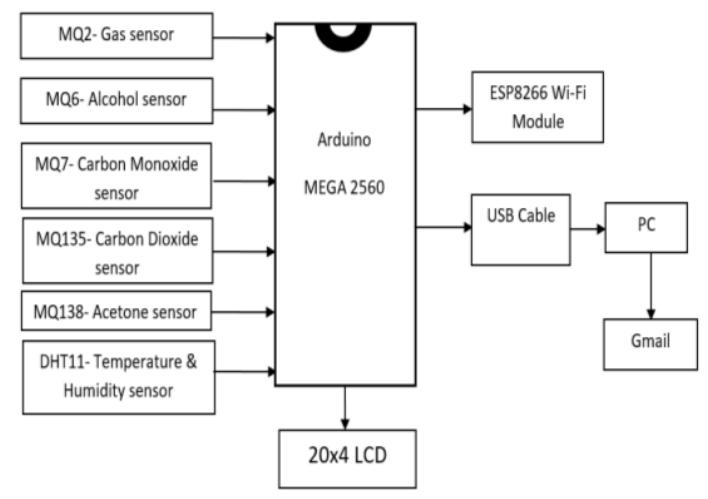

Figure 2. System design

Published By:

Blue Eyes Intelligence Engineering

\& Sciences Publication 


\section{STRUCTURE OF E-NOSE}

E-Nose gadget is utilized to distinguish the smell from the breath and perform investigation for recognizable proof. Here E-Nose is planned by

\begin{tabular}{|l|l|}
\hline \multicolumn{1}{|c|}{ Sensor } & \multicolumn{1}{c|}{ Operation } \\
\hline MQ-2 & Gas \\
\hline MQ-6 & Alcohol Gas \\
\hline MQ-7 & Carbon Monoxide (CO) \\
\hline MQ-135 & Carbon Dioxide (CO2) \\
\hline MQ-138 & Acetone \\
\hline DHT11 & Temperature \& Humidity \\
\hline
\end{tabular}

Table 1. List of gas sensors

utilizing six sensors installed in the veil and Arduino MEGA 2560 alongside a WIFI module

and SD card to store information for further use. The ENose can be found in Figure 3. Rundown of Gas sensors can be found in TABLE 1 .

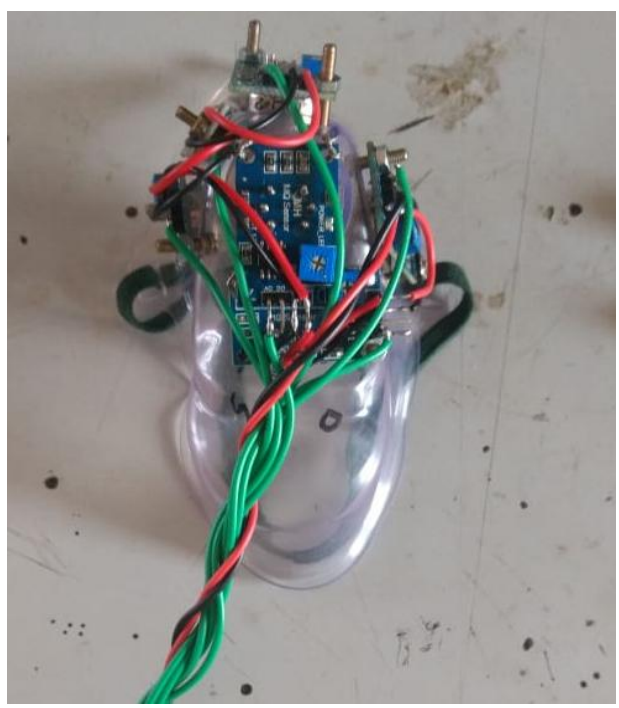

Figure 3. E-nose

The sensors utilized in E-Nose are simple sensors and consequently it requires an equation to change over the simple qualities to computerized esteems (ADC) in parts per million (ppm). To compute the sensor obstruction (Rs), we have the equation (1) and (2).

$$
\begin{aligned}
& R S=\frac{V c-V R L}{V R L} \chi R L \\
& V R L=\frac{A D C \times V c}{1023}
\end{aligned}
$$

Where, $\mathrm{Vc}$ is the voltage of arduino board, VRL is the voltage of sensor in test space and RL is the heap opposition estimated by $\Omega$ meter. ADC is the yield of the sensor in advanced qualities. Rs is the crude yield from sensor. Crude yield is changed over into ppm by the equation (3) and (4).

$$
\begin{aligned}
& \mathrm{C}=\gamma\left[\frac{R s}{R o}\right]^{\tau}, \gamma, \tau, \in R^{+} \\
& \mathrm{c}=10 \frac{\log \left(\frac{\frac{R s}{R o}-\gamma}{\alpha}\right)}{\beta}, \alpha, \beta, \gamma
\end{aligned}
$$

$\mathrm{C}$ is the evaluated gas focus in ppm, Rs is the genuine sensor opposition and Ro is the sensor obstruction in clean air. $\alpha, \beta, \gamma, \tau$ are consistent values is the assessed gas fixation in ppm, Rs is the real sensor obstruction and Ro is the sensor opposition in clean air. $\alpha, \beta, \gamma, \tau$ are consistent qualities[3].

\section{ACQUISATION OF DATA}

Information procurement is performed to get information preparing set and feed into the classifier. There are 20 information put away in database in that 10 solid and 10 diabetic patient information are accessible. Blood glucose level underneath $120 \mathrm{mg} / \mathrm{dL}$ are viewed as sound or more $150 \mathrm{mg} / \mathrm{dL}$ are viewed as diabetic. Every patient breath test information was gathered for around $120 \mathrm{sec}$ utilizing ENose and qualities are shown in the LCD associated with arduino board. The reaction information from sensor in ppm are sent in one bundle by USB link to the Laptop in comma isolated esteem (csv) in one line for like clockwork and put away in SD card[4].

\section{SELECTIVE FEATURES}

Highlight determination is performed to expel excess issue as a result of connected qualities. The principle motivation behind component determination is to acquire ideal incentive from the variety of gas sensors for computation. While testing initial two qualities are prohibited to maintain a strategic distance from mutilation because of gas atoms in nature[6].

\section{CLASSIFICATION}

In the arrangement, we group the outcome utilizing the one class SVM classifier. After element determination, the removed qualities are given as contributions to SVM. One class SVM is utilized to distinguish objects of a particular class among all articles, by fundamentally gaining from a preparation set containing just the objects of that class. One class SVM will break down the information and characterize the outcome as either ordinary or diabetic[5].

\section{TESTING \& RESULTS}

Testing was done on two classes of individuals to gauge how basic the E-Nose is utilized for ongoing reason. Testing were performed on typical and diabetic people. At first the outcomes were acquired in Matlab alongside the diagram . Further the outcomes will be sent to patient's mail ID. The acquired outcomes can likewise be seen as a Graphical portrayal on the made Web page. For future reference the information are put away in the Memory card 


\section{CONCLUSION}

This paper proposes a non-intrusive breath examination framework that identifies diabetes. Testing was performed to separate ordinary and diabetic patients utilizing unassuming component extraction and choice. Here we utilize straightforward Machine Learning calculation so preparing can be performed with no limitations. On further examinations bigger number of information can be gathered and prepared to obtain more precision. This framework adds to a straightforward non-obtrusive diabetes screening approach that gives comfortness to the patient.

\section{REFERENCES}

1. Rogers PH, Benkstein KD, Semancik S. Machine learning applied to chemical analysis: Sensing multiple biomarkers in simulated breath using a temperaturepulsed electronic-nose. Analytical chemistry. 2012 Nov 6;84(22):9774-81.

2. Saraoğlu HM, Selvi AO, Ebeoğlu MA, Taşaltin C Electronic nose system based on quartz crystal microbalance sensor for blood glucose and $\mathrm{HbAlc}$ levels from exhaled breath odor. IEEE Sensors Journal. 2013 Nov;13(11):4229-35.

3. Fens N, Van der Schee MP, Brinkman P, Sterk PJ. Exhaled breath analysis by electronic nose in airways disease. Established issues and key questions. Clinical \& Experimental Allergy. 2013 Jul;43(7):705-15.

4. Sarno R, Wijaya DR. Detection of diabetes from gas analysis of human breath using e-Nose. In2017 11th International Conference on Information \& Communication Technology and System (ICTS) 2017 Oct 31 (pp. 241-246). IEEE.

5. Castro M, Kumar B, Feller JF, Haddi Z, Amari A, Bouchikhi BE. Novel e-nose for the discrimination of volatile organic biomarkers with an array of carbon nanotubes (CNT) conductive polymer nanocomposites (CPC) sensors. Sensors and Actuators B: Chemical. 2011 Nov 28;159(1):213-9.

6. Saraoglu HM, Kocan M. Determination of blood glucose level-based breath analysis by a quartz crystal microbalance sensor array. IEEE Sensors Journal. 2009 Dec 11;10(1):104-9. 